\author{
Hans U. Rothen \\ Kay Stricker \\ Johanna Einfalt \\ Peter Bauer \\ Philip G. H. Metnitz \\ Rui P. Moreno \\ Jukka Takala
}

\section{Variability in outcome and resource use in intensive care units}

Received: 19 September 2006

Accepted: 24 April 2007

Published online: 1 June 2007

(C) Springer-Verlag 2007

\section{Electronic supplementary material}

The online version of this article

(doi:10.1007/s00134-007-0690-3) contains supplementary material, which is available to authorized users.

This research was supported financially by the European Society of Intensive Care Medicine (ESICM), Portuguese Society of Intensive Care (SPCI), Austrian Centre for Documentation and Quality Assurance in Intensive Care Medicine (ASDI), iMDsoft Corp., and Fund of the Austrian National Bank (project 10995 ONB).

This article is discussed in the editorial available at: http://

dx.doi.org/10.1007/s00134-007-0697-9.

H. U. Rothen ( $\bullet$ ) J. Takala

University Hospital, Department of Intensive Care Medicine,

Murtenstrasse 35, 3010 Berne, Switzerland e-mail: hrothen@insel.ch

Tel.: +41-31-6321176

Fax: +41-31-6329644

\section{K. Stricker}

University Hospital, Department of

Anesthesiology and Department of Intensive Care Medicine,

3010 Berne, Switzerland
J. Einfalt $\cdot$ P. Bauer

University of Vienna, Department of

Medical Statistics,

Vienna, Austria

\section{P. G. H. Metnitz}

University Hospital of Vienna, Department of Anesthesiology and General Intensive

Care,

Vienna, Austria

\section{R. P. Moreno}

Hospital de St. António dos Capuchos, Centro Hospitalar de Lisboa (Zona Central), Unidade de Cuidados Intensivos Polivalente, Lisbon, Portugal

\section{Abstract Objective: To examine variability in outcome and resource} use between ICUs. Secondary aims: to assess whether outcome and resource use are related to ICU structure and process, to explore factors associated with efficient resource use. Design and setting: Cohort study, based on the SAPS 3 database in 275 ICUs worldwide. Patients: 16,560 adults. Measurements and results: Outcome was defined by standardized mortality rate (SMR). Standardized resource use (SRU) was calculated based on length of stay in the ICU, adjusted for severity of acute illness. Each unit was assigned to one of four groups: "most efficient" (SMR and
SRU < median); "least efficient" (SMR, SRU > median); "overachieving" (low SMR, high SRU), "underachieving" (high SMR, low SRU). Univariate analysis and stepwise logistic regression were used to test for factors separating "most" from "least efficient" units. Overall median SMR was 1.00 (IQR 0.77-1.28) and SRU 1.07 (0.76-1.58). There were 91 "most efficient", 91 "least efficient", 47 "overachieving", and 46 "underachieving" ICUs. Number of physicians, of full-time specialists, and of nurses per bed, clinical rounds, availability of physicians, presence of emergency department, and geographical region were significant in univariate analysis. In multivariate analysis only interprofessional rounds, emergency department, and geographical region entered the model as significant. Conclusions: Despite considerable variability in outcome and resource use only few factors of ICU structure and process were associated with efficient use of ICU. This suggests that other confounding factors play an important role.

Keywords Intensive care unit - Severity of illness - Resource use - ICU mortality $\cdot$ Health services research . Quality assessment 


\section{Introduction}

In recent years organizational issues and quality management have become an important focus of interest [1]. Accordingly the assessment of resource use and outcome today is an integral part of critical care medicine. As a consequence there is a need for an extended analysis of the link between ICU structures and the process of care, on the one hand, and resource use and outcome, on the other. In general it is thought that there is marked variability between individual intensive care units (ICUs), both with respect to outcome and with respect to resource use $[2,3]$. However, only limited information exists about factors influencing such variability $[4,5]$. Most studies in this field are limited to a subset of patients, often defined by primary diagnosis, or include patients from only one country. For example, the ICU nurse-to-patient ratio has been shown to be associated with morbidity, hospital mortality, and resource use after esophagectomy [6] and with the risk of complications after abdominal aortic surgery [7]. In a general sample from the Austrian database variation in mortality was present even after adjustment for severity of illness, and this was explained at least in part by differences in case mix and standards of care [8]. Further factors relevant for such variation in outcome may include pre-ICU care, ICU staff workload [9], occupancy rate and volume of activity [10-12], and variations in post-ICU standards of care. Whether other factors, such as organizational characteristics of the hospital and ICU, type and size of the ICU, and variables related to physician staffing, are associated with variability in resource use and outcome has not been studied extensively.

As part of the recently presented SAPS 3 project $[13,14]$, apart from detailed information on individual patient outcome, data reflecting ICU structure and process were collected for each participating unit. A considerable number of units from around the world participated in the project, providing a unique opportunity to analyze the relationships between resource use, outcome, and ICU characteristics in a large sample of ICUs encompassing a broad range of structures, patient processes, and geographical regions, and based on standardized data collection.

The primary aim of this study was to examine whether there is wide variability in outcome and resource use between individual ICUs worldwide, even controlling for acute illness on admission to the ICU. Secondary aims were to test the hypothesis that outcome and resource use are related to ICU structure and process and to explore factors associated with efficient resource use as defined by low resource use per surviving patient. Part of this material has been presented as abstract [15].

\section{Materials and methods}

Database

This study is based on the database collected for the SAPS 3 study $[13,14]$. Of the cohort used to calculate the SAPS 3 score we included 275 of 303 ICUs with a total of 16,560 out of 16,784 patients. A detailed description of the study cohort, including information on data collection and data quality, has been presented previously [13]. For analysis in the present study patients who were readmitted to the ICU during the hospital stay were excluded. Further, only ICUs which had collected data for at least one surviving patient were included. Approval from the local ethics or data-protection committees were obtained as described previously $[13,14]$.

\section{Outcome and resource use}

Outcome was assessed using standardized mortality rate (SMR) defined as the quotient of observed to predicted mortality, and the SAPS 3 admission score was used to predict vital status at hospital discharge $[14,16]$. To assess resource use standardized, severity-adjusted resource use (SRU) was calculated for each individual ICU. We used SRU to estimate the average amount of resources used per surviving patient in a specific ICU. Of the many surrogate parameters that may be used to estimate resource use only length of stay in the ICU (LOS-ICU) was systematically collected for all patients in the SAPS 3 project. Based on these data the expected, severity-adjusted resource use per surviving patient was calculated as follows: In a first step the aggregate data were stratified according to SAPS 3 admission score. For each stratum the sum of LOS-ICU of all patients in that stratum was calculated. Finally, this sum was divided by the number of surviving patients, resulting in the average number of days in ICU used per surviving patient in the respective stratum (see Table 1). Units with no surviving patient were thus not included in this analysis (see also above). For example, in SAPS 3 class 1 the total LOS-ICU of all patients is 1,171 days, and the total number of surviving patients is 517 , resulting in an average length of stay of 2.3 days; thus in SAPS 3 class 1 the "expected resource use" per surviving patient is 2.3 days. To calculate SRU for a specific ICU (called "unit A" in this example), "expected resource use" per stratum was first multiplied by the total number of surviving patients of unit A in that stratum. This product is the expected total number of days that the "average" ICU would use to "produce" the number of surviving patients observed for unit A. The sum of the expected number of days of all strata 
Table 1 Length of stay ( $L O S)$ per surviving patient, stratified by SAPS 3. $L O S_{\text {total }}$ is the sum of all LOS within the respective SAPS 3 stratum. LOS $O S_{\text {per survivor }}$ is calculated as $L O S_{\text {total }} /$ number of surviving patients

\begin{tabular}{lrrrrrr}
\hline $\begin{array}{l}\text { SAPS } \\
\text { class }\end{array}$ & Points & Range & Mean \pm SD & \multicolumn{2}{c}{ No. of patients } & \multicolumn{2}{c}{ LOS (days) } \\
& Total & Surviving & Total & Per survivor \\
\hline 1 & $0-24$ & $21.4 \pm 2.6$ & 518 & 517 & 1,171 & 2.3 \\
2 & $25-34$ & $30.5 \pm 2.8$ & 2,516 & 2,494 & 7,914 & 3.2 \\
3 & $35-44$ & $39.7 \pm 2.8$ & 4,056 & 3,930 & 17,064 & 4.3 \\
4 & $45-54$ & $49.4 \pm 2.9$ & 3,667 & 3,284 & 23,664 & 7.2 \\
5 & $55-64$ & $59.1 \pm 2.9$ & 2,750 & 2,125 & 23,283 & 11.0 \\
6 & $65-74$ & $69.1 \pm 2.8$ & 1,689 & 968 & 16,090 & 16.6 \\
7 & $75-84$ & $78.8 \pm 2.8$ & 855 & 358 & 7,593 & 22.2 \\
8 & $85-94$ & $88.7 \pm 2.8$ & 359 & 102 & 3,000 & 29.4 \\
9 & 95 & $101.9 \pm 6.4$ & 174 & 31 & 1,208 & 39.0 \\
\hline
\end{tabular}

( $\sum$ LOS-ICU $\left.\mathrm{U}_{\text {expected }}\right)$ represents the severity-adjusted total sum of days which the "average" ICU would use to "produce" the number of surviving patients observed for unit A. Finally, SRU for unit A is defined as the sum of actual, observed LOS-ICU of all patients in that unit

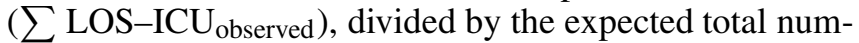
ber of days of all strata of that unit ( $\sum$ LOS-ICU $\left.\mathrm{I}_{\text {expected }}\right)$ : $\mathrm{SRU}=\sum$ LOS-ICU $\mathrm{Ibserved} / \sum$ LOS-ICU $\mathrm{ICxpected}_{\text {. Ac- }}$ cordingly, SRU reflects the severity-adjusted, observed to expected ratio of resources (estimated as length of stay in the ICU) used by a specific unit. Units with high SRU use a larger amount of resources to have a certain amount of surviving patients, as compared to units with low SRU. Based on median SMR and median SRU, each unit was assigned to one of four groups: Group 1 ("most efficient") included all units whose SMR and SRU were below the median SMR and SRU, respectively; group 2 ("least efficient") included units in which SMR and SRU were both above the median. Group 3 ("overachieving" or relatively wasteful: low SMR but high SRU) and group 4 ("underachieving": high SMR but low SRU) were defined accordingly (Fig. 1). Similarly, the units also were divided into nine groups using tertiles of SMR and SRU (Fig. 1). This latter was used to test for stability of our results, looking at the more "extreme" units only.

\section{ICU structure and process}

The following measures of structure were available from the SAPS 3 database: type of hospital (university vs. nonuniversity), presence of an emergency department in the hospital, number of staffed ICU beds, number of nurses per bed, total number of physicians per bed (includes all physicians assigned to the ICU), number of intensive care medicine (IM) physicians (i.e., physicians trained in intensive care medicine) per bed, and total number of different specialties available in the hospital. As measures of process, the following items were included: presence of multidisciplinary meetings, presence of inter-professional clinical rounds (i.e., participation of both physicians and nurses), availability of physicians in the ICU on weekdays,

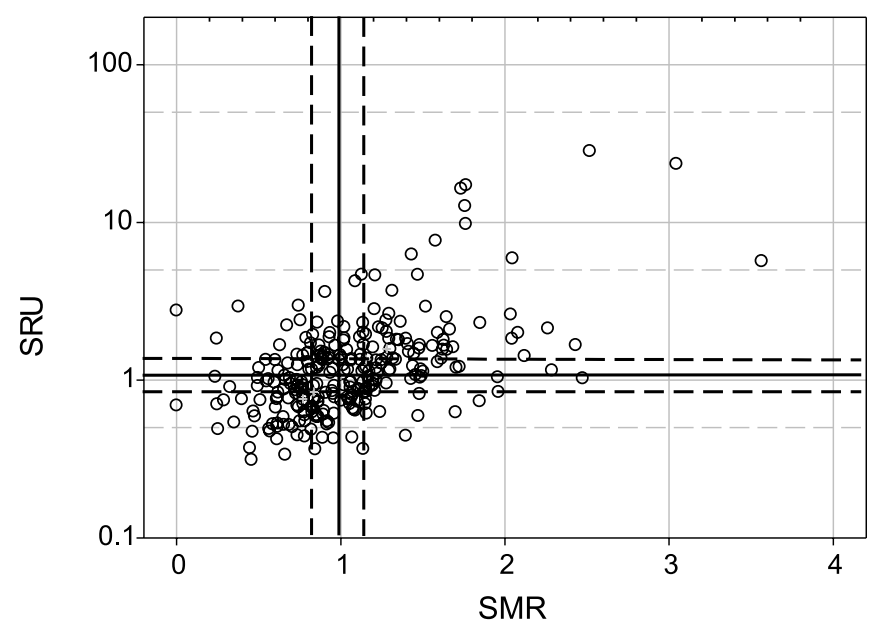

Fig. 1 Standardized mortality ratio $(S M R)$ and standardized resource use $(S R U)$. SMR is defined as observed/expected mortality ratio. SMR values higher than 1 (median SMR) represent "above average" mortality ratio (for calculation of SRU see text); SRU values higher than 1.07 (median) represent "above average" resource use per surviving patient. Unbroken lines Stratification by median; dashed lines Stratification by tertiles

and availability of physicians during the night and on weekends. Further details on the above variables can be found elsewhere [13, 14]. In addition, the geographic location of each ICU was available for further analysis. Based on the work presented previously [13, 14], each ICU was assigned to one of seven regions: Australasia, Central and South America, Central and Western Europe, Eastern Europe, Southern Europe and Mediterranean countries, Northern Europe, and North America. As the number of countries for the latter two regions was small, they were included in the region "Central and Western Europe" for the present analysis. A few additional variables collected for the SAPS 3 project were excluded from further analysis because too many data $(>10 \%)$ were missing. Definitions of all concepts were provided in advance, either online (for participants using the Internet version), as a help file (for those using the stand-alone version), or on paper $[13,14]$. 
Statistical analysis

Statistical analysis was performed using SAS for Windows versions 8 and 9 (SAS Institute, Cary, N.C., USA). A $p$ value less than 0.05 was considered statistically significant Unless stated otherwise, data are presented as mean \pm SD (median/interquartile range). To study relationships between variables Pearson's correlation coefficient was calculated. Univariate logistic regression analyses were used to test for factors of ICU structure and process that might allow separating "most efficient" from "least efficient" ICUs. Finally, variables that were significant in the univariate analyses were included in multiple, forwardbackward stepwise logistic regression analyses [17]. For this procedure effects are entered and removed in such a way that each forward selection step (applying a twotailed significance level of 0.05 ) may be followed by one or more backward elimination steps (also at the two-tailed significance level of 0.05). The variation explained by the model was estimated by calculating $R^{2}$ according to the methods of Cox and Snell and of Nagelkerke. To assess discriminative capability the area under the receiver operating characteristic curve was calculated. In all logistic regressions the "most efficient" ICUs have been predicted.

\section{Results}

Overall the median standardized mortality ratio (SMR) was 1.00 and median standardized resource use (SRU) was 1.07. The 10th and 90th percentiles were 0.60 and 1.63 for SMR, and 0.55 and 2.34 for SRU. For further details see Table 2. Summary measures of key variables for structure and process for these units are shown in the Electronic Supplementary Material (ESM, Table 2E). There were 228 units providing 20 or more patients for further analysis. For these units SMR was $1.01 \pm 0.36(0.96 / 0.77-1.21)$ and SRU was $1.27 \pm 1.91(0.98 / 0.73-1.41)$. The 10th and 90th percentiles were 0.61 and 1.48 for SMR and 0.53 and 1.93 for SRU. For the 133 units with more than 50 patients SMR was $0.95 \pm 0.36(0.86 / 0.74-1.14)$ and
SRU $0.99 \pm 0.52(0.87 / 0.65-1.22)$. The 10th and 90th percentiles were 0.56 and 1.48 for SMR and 0.50 and 1.61 for SRU. Figure 1 shows both SMR and SRU for each individual ICU. Using stratification by median SMR and SRU, there were 91 "most efficient" and 91 "least efficient" ICUs, as well as 47 "overachieving" and 46 "underachieving" units. There was a positive relationship between SMR and SRU $\left(R=0.46, R^{2}=0.21\right)$. Summary of key variables, stratified by "most efficient" and "least efficient" ( $<$ median/lowest tertile and $>$ median/highest tertile, respectively) are shown in Table 2 , and Table 3 summarizes key variables with stratification by region. Further, Figs. 2E and 3E (ESM) show SMR and SRU stratified by region, type of hospital, presence of emergency department in the hospital, and presence of common clinical rounds in the ICU.

The results of univariate and stepwise logistic regression analyses for factors of ICU structure and process that may allow differentiation of "most efficient" from "least efficient" ICUs (using median) are shown in Tables 4 and 5. Of all available variables representing ICU structure and process the following were significant in univariate analysis: number of physicians per bed, number of full-time specialists per bed, number of nurses per bed, number of physicians per nurse, presence of common clinical rounds, availability of a physician on weekdays, availability of a physician on weekends, presence of an emergency department in the hospital, and region. In the multivariate analysis, however, only presence of interdisciplinary clinical rounds, presence of an emergency department in the hospital, and region entered the model as significant. Thus, if interprofessional clinical rounds are held in a unit, there is a 2.7 times (95\% CI 1.2-6.2) higher chance that this unit belongs to the group of "most efficient" ICUs rather than to the group of "least efficient" ICUs. Similarly, presence of an emergency department results in a 7.0 times (95\% CI 1.3-37.1) higher chance of belonging to the group of "most efficient" units. On the other hand, variables related to nursing or physician staffing did not influence assignment of a unit to one of these groups. Finally, units located in Central and
Table 2 Stratification of ICUs by estimated efficiency: summary data; data are median (25th-75th percentiles) where appropriate (SMR standardized mortality ratio, SRU standardized resource use,

\begin{tabular}{llllll}
\hline SMR and SRU & $\begin{array}{l}\text { Most efficient } \\
\text { < Lowest tertile }\end{array}$ & < Median & $\begin{array}{l}\text { Least efficient } \\
>\text { Median }\end{array}$ & A Highest tertile & All units \\
\hline No. of units & 45 & 91 & 91 & 53 & 275 \\
No. of patients & 4,416 & 7,846 & 3,858 & 1,824 & 16,560 \\
SMR & $0.65(0.51-0.77)$ & $0.74(0.60-0.84)$ & $1.34(1.16-1.63)$ & $1.52(1.31-1.76)$ & $1.00(0.77-1.28)$ \\
SRU & $0.60(0.50-0.70)$ & $0.70(0.54-0.87)$ & $1.67(1.34-2.30)$ & $1.99(1.64-2.92)$ & $1.07(0.76-1.58)$ \\
Physicians per bed & $0.74(0.39-1.00)$ & $0.75(0.41-1.05)$ & $0.81(0.50-1.08)$ & $0.88(0.48-1.14)$ & $0.81(0.50-1.13)$ \\
Nurses per bed & $3.36(2.50-4.20)$ & $3.18(2.47-4.18)$ & $3.00(2.17-3.60)$ & $3.00(1.86-3.88)$ & $3.00(2.33-3.88)$ \\
IM specialists per bed & $0.28(0.13-0.50)$ & $0.29(0.14-0.58)$ & $0.33(0.25-0.70)$ & $0.33(0.24-0.71)$ & $0.37(0.18-0.74)$ \\
Physicians per nurse & $0.19(0.13-0.30)$ & $0.22(0.13-0.37)$ & $0.28(0.18-0.46)$ & $0.32(0.21-0.47)$ & $0.28(0.17-0.39)$ \\
\hline
\end{tabular}

IM intensive care medicine, All units data for the overall sample included in this study) 
Table 3 Variables by geographical region; data are median (25th75th percentiles) where appropriate (Region 1 Australasia, Region 2 Central + South America, Region 3 Central + Western + Northern
Europe + North America, Region 4 Eastern Europe, Region 5 Southern Europe + Mediterranean Region, SMR standardized mortality ratio, $S R U$ standardized resource use, $I M$ intensive care medicine)

\begin{tabular}{llllll}
\hline & Region 1 & Region 2 & Region 3 & Region 4 & Region 5 \\
\hline No. of units & 14 & 39 & 60 & 26 & 136 \\
No. of patients & 1,734 & 2,097 & 5,232 & 860 & 6,637 \\
SMR & $0.84(0.65-1.09)$ & $1.34(0.93-1.65)$ & $0.86(0.69-1.15)$ & $1.07(0.83-1.21)$ & $0.99(0.77-1.24)$ \\
SRU & $0.62(0.50-1.03)$ & $1.30(0.89-1.56)$ & $0.84(0.58-1.16)$ & $1.14(0.73-2.35)$ & $1.19(0.86-1.79)$ \\
Physicians per bed & $0.59(0.43-0.79)$ & $0.94(0.48-1.43)$ & $0.58(0.36-1.00)$ & $0.60(0.36-1.00)$ & $0.88(0.63-1.26)$ \\
Nurses per bed & $4.29(3.20-4.73)$ & $2.10(0.90-3.14)$ & $3.80(2.86-5.08)$ & $2.75(2.00-3.86)$ & $3.00(2.33-3.33)$ \\
IM specialists per bed & $0.18(0.14-0.29)$ & $0.26(0.13-0.86)$ & $0.21(0.13-0.33)$ & $0.29(0.17-0.50)$ & $0.55(0.38-0.83)$ \\
Physicians per nurse & $0.15(0.13-0.18)$ & $0.47(0.31-0.80)$ & $0.16(0.09-0.24)$ & $0.28(0.13-0.37)$ & $0.31(0.23-0.45)$ \\
\hline
\end{tabular}

Table 4 Estimated efficiency: univariate logistic regression analysis of explanatory variables. Comparing groups 1 ("most efficient") and 2 ("least efficient"). For details see text. Type of hospital: $Y=$ university hospital, $N=$ nonuniversity hospital. Emergency department $(E D): Y=$ hospital has an $\mathrm{ED}, N=$ hospital has no ED. Clinical rounds: $Y=$ clinical rounds (physicians and nursing staff together) in the ICU, $N=$ no common clinical rounds in the ICU. Physicians (weekdays): $Y=$ physicians are available in ICU on weekdays, $N=$ physicians are not available. Physicians (nights/weekends): $Y=$ physicians are available in ICU during the night and/or on weekends, $N=$ physicians are not available. Region: see also legend to Table 3 [OR odds ratio for being in the "most efficient" group as compared to the "least efficient" group (for region: OR is compared to region 3 as reference group), $C I$ confidence interval]

\begin{tabular}{|c|c|c|c|}
\hline & OR & $95 \% \mathrm{CI}$ & $p$ \\
\hline Number of staffed beds & 1.02 & $0.98-1.05$ & 0.351 \\
\hline Physicians per bed & 0.49 & $0.26-0.92$ & 0.026 \\
\hline $\begin{array}{l}\text { Number of intensive care } \\
\text { medicine specialists }\end{array}$ & 0.96 & $0.90-1.02$ & 0.164 \\
\hline $\begin{array}{l}\text { Intensive care medicine } \\
\text { specialists per bed }\end{array}$ & 0.29 & $0.11-0.75$ & 0.010 \\
\hline $\begin{array}{l}\text { Number of medical } \\
\text { specialties in hospital }\end{array}$ & 1.03 & $0.95-1.11$ & 0.497 \\
\hline Nurses per bed & 1.30 & $1.02-1.65$ & 0.035 \\
\hline Physicians per nurse & 0.03 & $0.00-0.22$ & $<0.001$ \\
\hline Type of hospital & 0.85 & $0.45-1.60$ & 0.608 \\
\hline $\begin{array}{l}\text { Emergency department } \\
\text { in hospital }\end{array}$ & 4.43 & $1.18-16.65$ & 0.027 \\
\hline Multidisciplinary meetings & 1.16 & $0.61-2.21$ & 0.647 \\
\hline Clinical rounds & 4.34 & $2.17-8.64$ & $<0.001$ \\
\hline Physicians (weekdays) & 0.31 & $0.13-0.70$ & 0.005 \\
\hline Physicians (nights/weekends) & 0.34 & $0.15-0.79$ & 0.012 \\
\hline \multicolumn{4}{|l|}{ Region } \\
\hline 1 & 0.49 & $0.12-2.06$ & 0.333 \\
\hline 2 & 0.05 & $0.02-0.18$ & $<0.001$ \\
\hline 3 & 1.00 & - & - \\
\hline 4 & 0.11 & $0.02-0.61$ & 0.012 \\
\hline 5 & 0.09 & $0.03-0.25$ & $<0.001$ \\
\hline
\end{tabular}

South America had a 0.09 times (95\% CI 0.02-0.35) lower chance and units located in South Europe and Mediterranean areas had a 0.14 times (95\% CI 0.05-0.42) lower chance of belonging to the "most efficient" group rather than to the "least efficient" group (both compared to ICUs located in Northern, Central, or Western Europe or North America). Note that the variable "physicians
Table 5 Estimated efficiency: multiple regression analysis of explanatory variables. Comparing groups 1 ("most efficient") and 2 ("least efficient"); for details see text. Stepwise logistic regression analysis. Initially all significant variables of univariate analysis are entered into the model. For region, odds ratio $(O R)$ is compared to region 3 (reference group) ( $n a$ variable did not enter the model; for further legends see Table 4)

\begin{tabular}{llll}
\hline Variable & OR & $95 \%$ CI & $p$ \\
\hline $\begin{array}{l}\text { Physicians per bed } \\
\text { Intensive care medicine }\end{array}$ & na & - & - \\
specialists per bed & na & - & - \\
Nurses per bed & na & - & - \\
Physicians per nurse & na & - & - \\
Emergency department & & & \\
in hospital & 6.96 & $1.30-37.12$ & 0.023 \\
Clinical rounds & 2.70 & $1.18-6.19$ & 0.019 \\
Physicians (weekdays) & na & - & - \\
Physicians (nights/weekends) & na & - & - \\
Region & & - & - \\
$\quad 1$ & 0.42 & $0.09-1.92$ & 0.264 \\
2 & 0.09 & $0.02-0.35$ & 0.001 \\
3 & 1.00 & - & - \\
4 & 0.19 & $0.02-1.74$ & 0.14 \\
5 & 0.14 & $0.05-0.42$ & 0.001 \\
\hline
\end{tabular}

per nurse" was not included in multivariate analysis due to collinearity with the two other variables "physicians per bed" and "nurses per bed". Overall the model only explains a low proportion of variance with $R^{2}=0.24$ (Cox and Snell), $R^{2}=0.33$ (Nagelkerke), and has a moderate discriminative capability with an area under the receiver operating characteristics curve of 0.70 . Including all interactions between the variables with a significant univariate contribution did not change the results since no interaction entered the model. Results of the univariate and stepwise logistic regression analyses, with the ICUs separated by tertiles, are presented in the ESM (Tables 4E and 5E).

\section{Discussion}

This study confirms marked variability in outcome and resource use in a large sample of intensive care units. Interestingly, of the many variables that were considered rep- 
resentative of ICU structure and process, and that could be included in our analysis, only the presence of interprofessional clinical rounds and of an emergency department in the hospital allowed separation of "most efficient" from "least efficient" intensive care units. In addition, the geographical region in which the ICU was located was identified as explanatory variable. This suggests that other ICUrelated and non-ICU-related factors, not included in the present study but differing between various regions or possibly countries, are relevant for outcome and resource use in intensive care medicine.

Although not designed to assess the efficiency of participating intensive care units, the SAPS 3 database $[13,14]$ provides a unique opportunity to analyze variability in outcome and resource use and factors influencing such variability in a large sample of ICUs [5]. To assess ICU performance four domains have been proposed [18]: medical outcomes, economic outcomes, psychosocial and ethical outcomes, and institutional outcomes. Of these, data on severity-adjusted survival rate are readily available in SAPS 3 as markers of medical outcomes. While interpreting this parameter is not without limitations, its use is widely accepted as it helps to identify variations in the process of care [19].

Assessing ICU performance solely based on medical outcomes and without taking into consideration resource use (a marker of economic outcome) may be biased [20] As opposed to assessment of survival rate, however, assessment of resource use is far less straightforward [21], and various models have been presented [22, 23]. In addition, the Therapeutic Intervention Scoring System has been used as a surrogate marker of resource use in a number of studies [24, 25]. As none of these measures was included consistently in the entire SAPS 3 database, we chose to use length of stay in the ICU as surrogate. A similar approach was used previously [26], while others have further modified this methodology, introducing, for example, the notion of weighted LOS [27]. We used the unmodified LOS-ICU as this measure is straightforward and easy to collect, and because the level of intensity of care may not only markedly change between the first and second day of a stay in the ICU but very often shows much more complex patterns during the course of critical illness [28].

A further possibility would have been to calculate on a patient per patient basis the predicted LOS-ICU from patient characteristics and to compare this parameter with observed LOS-ICU. For example, a linear regression model to predict severity-adjusted LOS in the hospital has recently been presented [29]. Still, using such a model the skewed distribution of LOS-ICU must be taken into account. Similar approaches also have been used by others $[16,30,31]$. However, we were aiming rather at a composite variable (SRU) that includes information from length of stay of a group of patients and number of surviving patients of that group. Of note, we deliberately included nonsurviving patients in order to have SRU as a parameter reflecting the total resource use of an ICU. Even nonsurviving patients consume resources, and if a unit works "ineffectively" (or wastefully), it may use large amounts of resources (used for nonsurviving patients) without having an appropriate number of surviving patients. Accordingly we were not aiming at a model predicting LOS, but at a model assessing resource use for a group of patients. In our study we used SAPS 3 strata to define such groups. SRU may thus be considered as a rough indicator of the resources needed to "produce" surviving patients.

As in previous studies [16, 30], our findings demonstrate wide variability both in outcome and resource use within intensive care units. For example, the 90th percentile of SMR differs from the 10th percentile by a factor of 2.7. For SRU the corresponding figure is 4.3 (or 3.2 if only units collecting data of more than 50 patients are considered). In contrast to previous publications, however, the current study covers a rather large sample of more than 250 ICUs from all over the world. Further, the magnitude of variation both in risk-adjusted mortality and in risk-adjusted resource use is greater than in previously published studies $[16,30]$.

Univariate analysis suggests that the presence of a higher number of physicians results in a less efficient ICU. This puzzling result needs analysis in further studies. Still, only a small number of key measures of ICU structure and process could be identified by multivariate analysis as being significantly linked to the selected measures of performance. Specifically, neither measures of nurse staffing nor of physician staffing were found to be predictive parameters that might allow separation of "most efficient" units from "least efficient" units. This finding was confirmed by additional analysis including only the highest and lowest tertiles (supplemental electronic material), and is in contrast to a number of previous studies $[6,32,33]$. On the other hand, differences in intensivist-to-ICU bed ratios over a wide range of 1:7.5-1:15 were not associated with differences in ICU or hospital mortality [34], and only at a ratio of 1:15 was there an increase in resource use as estimated by LOS-ICU. Also, no consistent association between ICU volume and risk-adjusted mortality was found in a recent study [35].

A possible explanation for these contradictory results is differences in staff workload [9] that may not be captured by simple staff-to-bed or staff-to-patient ratios. Probably more specific measures such as the Therapeutic Intervention Scoring System should be analyzed per staff member to capture such differences. Also, organizational issues $[36,37]$ may be more relevant in this respect than the numbers of staff per bed or patient. The latter notion is corroborated in the present study by the finding that presence of an emergency department in the hospital and presence of teamwork (i.e., clinical rounds with participation of nurses and physicians) both were variables associated with measures of ICU performance. 
In addition to the elements discussed above, a number of other factors not recorded by our study may cause differences in ICU performance, such as differences in the preICU patient process, ICU admission and discharge policies, specific elements of ICU processes (e.g., communication skills of team members, presence at the bedside), hospital volume [11], and post-ICU care, including availability of high-dependency or intermediate care units. In this respect the presence of an emergency department (which by itself may affect patient mix) also might be considered as a surrogate marker for some of these factors. This also may explain why this variable was found to be significant in our analysis. Finally, the recently described marked differences in intensive care medicine training [38] possibly are relevant for variability in outcome and resource use as well.

Of note, geographical region of the ICU was identified as a relevant factor linked to the variability in the selected measures of performance. This suggests that, in addition to the above factors, there may be, for example, consistent differences in case mix, life-style (e.g., smoking habits, alcohol abuse, prevalence of obesity) [14], or genetics between regions that are not revealed by the parameters included in the SAPS 3 project.

Our study has several limitations. First, this is a secondary analysis of data collected for the SAPS 3 project. Although the quality of patient-related data was good, there was a small amount of missing ICU-related data. Also, the original SAPS 3 study was not conceived primarily to serve as the basis of an extended analysis of resource use in critical care. Second, although data from a large number of patients were collected, there were some units providing data of only a few patients. Accordingly, due to random variation key variables of performance in such units may be under- or overestimated. However, analysis of, for example, subgroups of ICUs separated by tertile instead of median shows that the results of our study are rather robust. Third, the appropriateness of SMR and SRU as benchmarks to assess ICU performance or efficiency may be questioned [39]. Until now no thorough validation of these measures has been presented. However, as no gold standard exists, and because "performance" in general is considered a multidimensional concept $[18,40]$, we consider our approach reasonable. Fourth, although SAPS 3 was developed using a broad array of clinical variables, there still is the possibility that unmeasured variables at the patient level may play an additional role. However, we propose that this probably is of minor importance, given the large clinical experience with previous versions of SAPS and the rigorous, detailed analysis used in the SAPS 3 project, including variables representing lead-time bias and case mix. Fifth, our study only addresses resource use in the ICU. It is clear that processes and decision making outside the ICU can have a major impact on the overall resource utilization. However, in this study no data were available to appropriately address this issue. Finally, the so-called "country coordinator" effect, in which country coordinators tend to choose similar ICUs, could have biased the sample. However, it is not probable that this effect played a role in such a large study.

\section{Conclusion}

In conclusion, despite considerable variability in outcome and resource use in this sample only a few factors of ICU structure and process were identified as being associated with efficient use of the ICU. This suggests that other confounding factors at the ICU level which were not included in this study play an important role.

Acknowledgements. We are grateful to all staff of participating ICUs who made this project possible. We express special thanks to Jeannie Wurz, Medical Writer/Editor, Department of Intensive Care Medicine, University Hospital Berne, Switzerland, for editing the manuscript and to Barbara Jordan, Department of Medical Statistics, University of Vienna, Vienna, Austria for support in statistical analyses.

\section{References}

1. Bion JF, Heffner JE (2004) Challenges in the care of the acutely ill. Lancet 363:970-977

2. Lassnigg A, Hiesmayr MJ, Bauer P, Haisjackl M (2002) Effect of centre-, patient- and procedure-related factors on intensive care resource utilisation after cardiac surgery. Intensive Care Med 28:1453-1461

3. Niskanen MM, Takala J (2001) Use of resources and postoperative outcome. Eur J Surg 167:643-649

4. Pollack MM, Patel KM (2002) Need for shift in focus in research into quality of intensive care. Lancet 359:95-96
5. Suter PM (2005) Some ICUs save more lives than others: we need to know why! Intensive Care Med 31:1301-1302

6. Amaravadi RK, Dimick JB, Pronovost PJ, Lipsett PA (2000) ICU nurse-to-patient ratio is associated with complications and resource use after esophagectomy. Intensive Care Med 26:1857-1862

7. Pronovost PJ, Dang D, Dorman T, Lipsett PA, Garrett E, Jenckes M, Bass EB (2001) Intensive care unit nurse staffing and the risk for complications after abdominal aortic surgery. Eff Clin Pract 4:199-206
8. Metnitz PG, Lang T, Vesely H, Valentin A, Le Gall JR (2000) Ratios of observed to expected mortality are affected by differences in case mix and quality of care. Intensive Care Med 26:1466-1472

9. Tucker J (2002) Patient volume, staffing, and workload in relation to risk-adjusted outcomes in a random stratified sample of UK neonatal intensive care units: a prospective evaluation. Lancet 359:99-107 
10. Iapichino G, Gattinoni L, Radrizzani D, Simini B, Bertolini G, Ferla L, Mistraletti G, Porta F, Miranda DR (2004) Volume of activity and occupancy rate in intensive care units. Association with mortality. Intensive Care Med 30:290-297

11. Kahn JM, Goss CH, Heagerty PJ, Kramer AA, O'Brien CR, Rubenfeld GD (2006) Hospital volume and the outcomes of mechanical ventilation. N Engl J Med 355:41-50

12. Glance LG, Li Y, Osler TM, Dick A, Mukamel DB (2006) Impact of patient volume on the mortality rate of adult intensive care unit patients. Crit Care Med 34:1925-1934

13. Metnitz PG, Moreno RP, Almeida E, Jordan B, Bauer P, Campos RA, Iapichino G, Edbrooke D, Capuzzo M, Le Gall JR (2005) SAPS 3-from evaluation of the patient to evaluation of the intensive care unit. I. Objectives, methods and cohort description. Intensive Care Med 31:1336-1344

14. Moreno RP, Metnitz PG, Almeida E, Jordan B, Bauer P, Campos RA, Iapichino G, Edbrooke D, Capuzzo M, Le Gall JR (2005) SAPS 3-from evaluation of the patient to evaluation of the intensive care unit. II. Development of a prognostic model for hospital mortality at ICU admission. Intensive Care Med 31:1345-1355

15. Rothen HU, Stricker KH, Einfalt J, Metnitz PG, Moreno RP, Takala J (2006) Variability in outcome and resource use in ICU's. Intensive Care Med 32(Suppl 1):S138

16. Rapoport J, Teres D, Lemeshow S, Gehlbach S (1994) A method for assessing the clinical performance and cost-effectiveness of intensive care units: a multicenter inception cohort study. Crit Care Med 22:1385-1391

17. Katz MH (2003) Multivariable analysis: a primer for readers of medical research. Ann Intern Med 138:644-650

18. Garland A (2005) Improving the ICU. I. Chest 127:2151-2164

19. Sirio CA, Shepardson LB, Rotondi AJ, Cooper GS, Angus DC, Harper DL, Rosenthal GE (1999) Community-wide assessment of intensive care outcomes using a physiologically based prognostic measure: implications for critical care delivery from Cleveland Health Quality Choice. Chest 115:793-801

20. Kaboli PJ, Barnett MJ, Fuehrer SM, Rosenthal GE (2001) Length of stay as a source of bias in comparing performance in VA and private sector facilities: lessons learned from a regional evaluation of intensive care outcomes. Med Care 39:1014-1024
21. Jegers M, Edbrooke DL, Hibbert CL, Chalfin DB, Burchardi H (2002) Definitions and methods of cost assessment: an intensivist's guide. Intensive Care Med 28:680-685

22. Negrini D, Sheppard L, Mills GH, Jacobs P, Rapoport J, Bourne RS, Guidet B, Csomos A, Prien T, Anderson G, Edbrooke DL (2006) International Programme for Resource Use in Critical Care (IPOC) - a methodology and initial results of cost and provision in four European countries. Acta Anaesthesiol Scand 50:72-79

23. Pronovost PJ, Needham DM, Waters $H$, Birkmeyer CM, Calinawan JR, Birkmeyer JD, Dorman T (2006) Intensive care unit physician staffing: financial modelling of the Leapfrog standard. Crit Care Med 34(Suppl 3):S18-S24

24. Clermont G, Angus DC, LindeZwirble WT, Lave JR, Pinsky MR (1998) Measuring resource use in the ICU with computerized therapeutic intervention scoring system-based data. Chest 113:434-442

25. Stricker K, Rothen HU, Takala J (2003) Resource use in the ICU: short- vs. long-term patients. Acta Anaesthesiol Scand 47:508-515

26. Angus DC, Linde-Zwirble WT, Sirio CA, Rotondi AJ, Chelluri L, Newbold RC 3rd, Lave JR, Pinsky MR (1996) The effect of managed care on ICU length of stay: implications for medicare. JAMA 276:1075-1082

27. Rapoport J, Teres D, Zhao Y, Lemeshow S (2003) Length of stay data as a guide to hospital economic performance for ICU patients. Med Care 41:386-397

28. Iapichino G, Radrizzani D, Bertolini G, Ferla L, Pasetti G, Pezzi A, Porta F, Miranda DR (2001) Daily classification of the level of care. A method to describe clinical course of illness, use of resources and quality of intensive care assistance. Intensive Care Med 27:131-136

29. Rosenthal GE, Harper DL, Quinn LM Cooper GS (1997) Severity-adjusted mortality and length of stay in teaching and nonteaching hospitals. Results of a regional study. JAMA 278:485-490

30. Render ML, Kim HM, Deddens J, Sivaganesin S, Welsh DE, Bickel K, Freyberg R, Timmons S, Johnston J, Connors AF Jr, Wagner D, Hofer TP (2005) Variation in outcomes in Veterans Affairs intensive care units with a computerized severity measure. Crit Care Med 33:930-939
31. Zimmerman JE, Kramer AA, McNair DS, Malila FM, Shaffer VL (2006) Intensive care unit length of stay: benchmarking based on Acute Physiology and Chronic Health Evaluation (APACHE) IV. Crit Care Med 34:2517-2529

32. Thorens JB, Kaelin RM, Jolliet P Chevrolet JC (1995) Influence of the quality of nursing on the duration of weaning from mechanical ventilation in patients with chronic obstructive pulmonary disease. Crit Care Med 23:1807-1815

33. Pronovost PJ, Angus DC, Dorman T, Robinson KA, Dremsizov TT, Young TL (2002) Physician staffing patterns and clinical outcomes in critically ill patients: a systematic review. JAMA 288:2151-2162

34. Dara SI, Afessa B (2005) Intensivist-tobed ratio: association with outcomes in the medical ICU. Chest 128:567-572

35. Durairaj L, Torner JC, Chrischilles EA, Vaughan Sarrazin MS, Yankey J, Rosenthal GE (2005) Hospital volumeoutcome relationships among medical admissions to ICUs. Chest 128:1682-1689

36. Carson SS, Stocking C, Podsadecki T, Christenson J, Pohlman A, MacRae S, Jordan J, Humphrey H, Siegler M, Hall J (1996) Effects of organizational change in the medical intensive care unit of a teaching hospital: a comparison of 'open' and 'closed' formats. JAMA 276:322-328

37. Baggs JG, Schmitt MH, Mushlin AI, Mitchell PH, Eldredge DH, Oakes D, Hutson AD (1999) Association between nurse-physician collaboration and patient outcomes in three intensive care units. Crit Care Med 27:1991-1998

38. Barrett H, Bion JF (2005) An international survey of training in adult intensive care medicine. Intensive Care Med 31:553-561

39. Thomas JW, Hofer TP (1999) Accuracy of risk-adjusted mortality rate as a measure of hospital quality of care. Med Care 37:83-92

40. Pronovost PJ, Nolan T, Zeger S, Miller M, Rubin H (2004) How can clinicians measure safety and quality in acute care? Lancet 363:1061-1067 